\title{
Spatiotemporal behaviors of the ridership of a public transportation system during an epidemic outbreak: case of MERS in Seoul
}

\author{
Ji-Hye Lee ${ }^{1} \cdot$ Segun $\mathrm{Goh}^{2} \cdot$ Jong Won $\mathrm{Kim}^{3} \cdot \mathrm{Keumsook} \mathrm{Lee}^{4} \cdot$ M. Y. Choi ${ }^{1}$
}

Received: 2 August 2021 / Revised: 21 August 2021 / Accepted: 23 August 2021 / Published online: 25 October 2021

(c) The Korean Physical Society 2021

\begin{abstract}
During May and June 2015, an outbreak of the Middle East respiratory syndrome (MERS) occurred in Korea, which raised the fear of contagion throughout society and suppressed the use of public transportation systems. Exploring daily ridership data of the Seoul bus transportation system, along with the number of infected patients and search volume in web portals, we observe that ridership decreased abruptly while attention was heavily focused online. Then this temporal reduction recovered exponentially with a characteristic time of 3 weeks when newly confirmed cases began to decrease. We also find with the data of ranked keywords of web portals that areas with severely reduced ridership tended to cluster and spatiotemporal variations of such clusters were highly associated with general hospitals where MERS patients were treated. On the other hand, the spatial reduction in ridership relaxed algebraically with the distance from a general hospital while the outbreak was severe. We further probe the influence of the epidemic outbreak in the framework of linear response theory, which relates the responses to the epidemic outbreak ("perturbation") with correlations in the absence of the perturbation. Indeed, the spatial correlation function of the ridership changes is observed to follow a power law, sharing the same exponent as the spatial relaxation of the response function. This new theoretical approach offers a useful tool for understanding responses of public transportation system to epidemic or accidental disasters.
\end{abstract}

Keywords Public transportation $\cdot$ Epidemic outbreak $\cdot$ Spatiotemporal pattern $\cdot$ Power law

\section{Introduction}

Public transportation is regarded as an important factor contributing to the rapid and extensive spread of infectious disease. For instance, in the beginning of the massive public

Keumsook Lee

kslee@sungshin.ac.kr

$\triangle$ M. Y. Choi

mychoi@snu.ac.kr

1 Department of Physics and Astronomy and Center for Theoretical Physics, Seoul National University, Seoul 08826, Korea

2 Theoretical Physics of Living Matter, Institute of Biological Information Processing, Forschungszentrum Jülich, 52425 Jülich, Germany

3 Department of Healthcare Information Technology, Inje University, Gimhae 50834, Korea

4 Department of Geography, Sungshin Women's University, Seoul 02844, Korea transport era, infected crews and passengers of ships and trains spread Spanish flu, which brought about a pandemic killing an estimated 20-50 million people in the year 1918 [1]. The prevalence of the severe acute respiratory syndrome (SARS) in the year 2002 showed how air travel contributed to the rapid spread of the newly emerging infection and could potentially even start a pandemic [2]. Most recently, the outbreak of novel coronavirus infection (COVID-19), having begun in China and spread rapidly over the world, also witnessed a striking instance of the key role of public transportation. On the other hand, public transportation is profoundly susceptible to epidemic outbreaks, especially in a metropolis, where public transportation accounts for a considerable percentage of passenger traffic and thus constitutes an integral part of everyday life. Newly emerging infections usually provoke restrictions in freight and passenger transport, which causes immediate and severe economic repercussions [3]. At an individual level, people's trip-making decisions have also been found to be influenced by epidemic outbreaks, resulting in trip rescheduling, re-routing, and 
cancellation [4-7]. Because many infectious diseases spread through various personal contacts, spatiotemporal behaviors, e.g., trip trajectories, of passengers are crucial for preventing or provoking a catastrophic outbreak [8-11]. Thus, a primary aim of our current study is to investigate changes in public transportation ridership (i.e., the number of passengers) induced by an epidemic outbreak, which could potentially provide valuable information for public health policies via a better understanding of epidemic dynamics.

Much research has been devoted to understanding the human movement under regular stationary circumstances, revealing the collective properties in daily activities of individuals [8, 12-21]. However, research on human movement under disturbed circumstances [11, 22-25], such as violent conflicts, life-threatening epidemic outbreaks, and other large-scale emergencies, still remains rather limited. In this regard, the outbreak of the Middle East respiratory syndrome (MERS) in the Republic of Korea during May and June 2015 provides an extraordinary opportunity to study public transportation in turmoil and to identify its social and economic impact [26, 27]. Note in particular that the outbreak in Korea was the largest one outside the Middle East and that it was localized mostly in Seoul, where about $2 / 3$ of all passengers used public transportation. On the other hand, COVID-19 is still ongoing in Seoul, as well as throughout the world, and as a result ridership data are actively being gathered. The study of the MERS case is also expected to provide a guide to analyzing the case of COVID-19 when it is subdued.

Like SARS and COVID-19, MERS is a respiratory infectious disease that spreads via close contact with infected persons. With symptoms including fever, cough, and shortness of breath, it is known to be a lethal epidemic [28]. Only 14 days after the first patient was confirmed on 20 May, the number of people diagnosed with the deadly disease jumped to 30 . The growth rate of the infected population climaxed on 7 June and became zero after 4 July. As of 28 July, the number of people diagnosed with MERS in the Republic of Korea was 186 and the death toll was 36 . The rapid spread and fatality of MERS evoked the fear of contagion throughout society and, thus, affected people's daily life. People tended to avoid outdoor activities and to shun public places [26]. This behavioral change induced an economic slowdown across the nation, including a reduction in the demand for tourism, entertainment, leisure, and dining [29]. The private education industry was also diminished due to the temporary shutdown of schools. It also adversely affected consumer expenditures [30]. In particular, public transport ridership, i.e., the number of passengers using public transport, was examined, and the MERS outbreak was reported to have resulted in a decrease in transit ridership in areas with relatively larger numbers of rail stations [31]; this result is in agreement with the report that the risk of the SARS infection was greater in Asian countries with high population densities [32]. A further report was that the impact of the MERS outbreak could be heterogeneous with respect to the start time, magnitude, and duration of avoiding public transportation [33]. The decrease in transport ridership at the peak time lasted for a very short period during the outbreak and proliferation of MERS while the decrease was greater and lasted longer in off-peak afternoons.

We use the ridership data of each stop in the Seoul bus transportation system to investigate the change induced by the MERS outbreak. Specifically, the system is considered to have been in equilibrium before the MERS outbreak and to have been perturbed by the outbreak. Then, the responses of the system to the perturbation are studied by means of linear response theory, which is a standard tool in statistical mechanics [34-37]. Most of all, it gives the response function in terms of the correlation function in equilibrium; this relation, known as the fluctuation-dissipation theorem, allows one to probe the response behavior, regardless of the detailed nature of the system and the perturbation. Relaxation to the stationary state and the corresponding behavior of the system are examined. The ridership in total is observed to decrease abruptly upon the outbreak and to relax exponentially with the characteristic time of 3 weeks. In particular, both the logarithmic change in the ridership and the spatial correlation function are found to exhibit power-law behaviors, in agreement with the fluctuation-dissipation theorem.

\section{Seoul bus transportation system and MERS outbreak}

The Seoul bus system is a major transportation mode in Metropolitan Seoul, accounting for more than $26 \%$ of the modal share in the year 2015 [38]. As of May 2015, there were 612 bus service routes and 15714 bus stops in total, among which 10588 stops were located in Seoul. More than 12,000,000 transactions were made on a weekday before the decrease caused by the MERS outbreak. The detailed information regarding the transactions is collected via smart cards, including the departure/arrival times and places tracked by the global positioning system. The smart-card data are managed by the Seoul City Government and not open to the public. With special permission, we have had access to the data, without any personal identification, collected from 17 to 23 May as well as from 3 to 17 June 2015. Processing these data together with the data on daily ridership of every stop without spatial information, open to the public from 2015 [39], we obtain extensive ridership data for several years. The total ridership data from 2015 to 2018 indicate similar seasonal trends every year. To avoid the seasonal effects on the comparison, we, thus, adopt the ridership data for 2016 


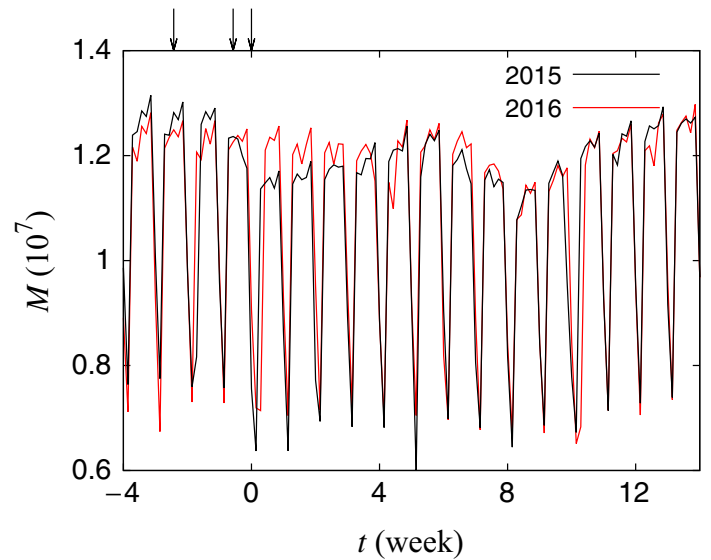

Fig. 1 Time evolution of the total ridership $M$ in the years 2015 (black) and 2016 (red). The first Saturday in June of each year, which is 6 June in 2015 and 4 June in 2016, is taken as the origin of time $(t=0)$. The three arrows mark the 3 days when the first infection (20 May), first death (2 June), and first cure (6 June) were officially reported in 2015. While the spiked dips manifest the weekly periodicity, the valley centered around $t=8$ reflects the summer vacation

as the reference. Note that the number of bus stops in 2015, matching those in 2016, is given by $N=7994$.

As shown in Fig. 1, the total ridership $M(t)=\sum_{\mathbf{r}} s(\mathbf{r} ; t)$, where $s(\mathbf{r} ; t)$ denotes the number of passengers using the stop at location $\mathbf{r}$ on given day $t$, exhibits weekly periodicity, obviously due to the sharp decrease on weekends [40]. In addition, small dips are observed on some weekdays, especially, Tuesdays and Thursdays, which presumably reflects the common lifestyle pattern of people who do not commute regularly. Here, the MERS outbreak induced a noticeable reduction in total ridership. The onset of MERS did not immediately affect the total ridership. While the first case of MERS in Korea was officially reported on 20 May 2015, the total ridership remained steady for 2 weeks thereafter, followed by an abrupt decrease. This decrease is distinctive in comparison with the data for the year 2016 because the total ridership in 2015 was, on average, larger than that in 2016 by $2 \%$. Indeed, the impact of the MERS outbreak was significant enough to destroy the above-mentioned weekly regularity in the total ridership. This sudden decrease was apparently triggered by the sequence of events on 2 June: The death, which had occurred the day before, was confirmed as the first death, and a second death as well as the first tertiary case was reported. The downtrend in total ridership lasted for 4 days due to the rising number of patients [see Fig. 2a for the number $m_{1}$ of new cases reported], as well as the paucity of information on the infection route. During the downtrend, the names of hospitals treating patients were not disclosed, so concerns among people resulted in an unceasing drop in the total ridership. The media extensively covered the events, and the concern over MERS was greatly heightened, as manifested by the Google search volume $m_{2}$

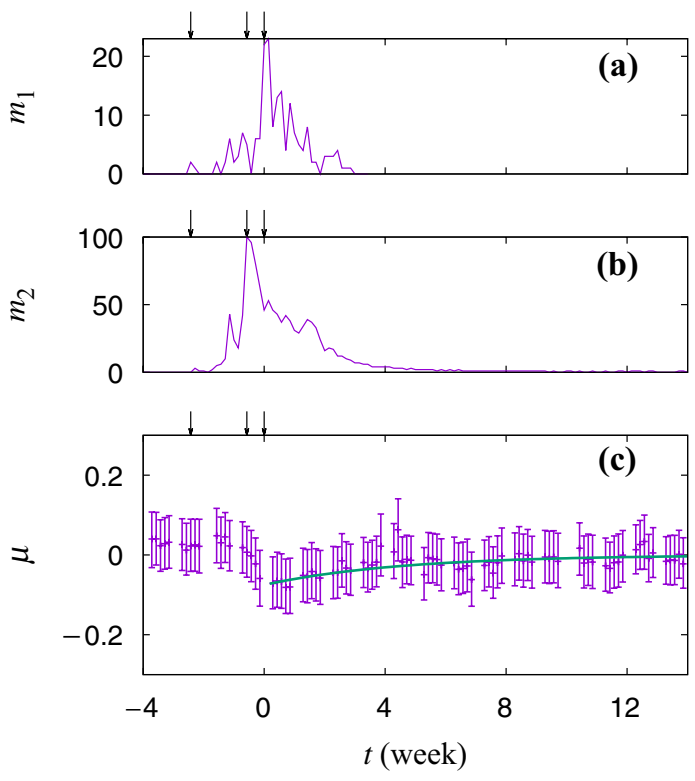

Fig. 2 a Daily number $m_{1}$ of new infections reported [41], b relative number $m_{2}$ of searches in Google [42], and c mean logarithmic change $\mu$, with error bars representing the first and the third quartiles, evolving in time $t$. In $\mathbf{c}$, data for weekends and holidays are omitted, and the blue line, which describes the exponential relaxation to zero with characteristic time $\tau \approx 3$ weeks [Eq. (4)], has been obtained via fitting the weekday data for 6 weeks (from 8 June to 17 July, i.e., $0 \leq t \leq 6$ ). The origin of time $t$ and the three arrows mark the same days as in Fig. 1

shown in Fig. 2b. After the first cured patient was reported on 6 June and the release of the names of the hospitals on 7 June, the total ridership gradually recovered from the influences of MERS. The weekly regularity was instantly restored. Comparison between 2015 and 2016 in Fig. 1 shows that the decrease was progressively alleviated except in the beginning of the summer vacation. (Note that the summer vacation lasts mostly from mid-July to mid-August in Korea.)

The ridership in a public transportation system varies greatly stop by stop, and its distribution usually assumes a nonGaussian form such as log-normal or Weibull form, characterized by skewness and heavy tails [43]. While the multiplicative Yule-type process is inherent for the temporal fluctuations of the ridership, correlations between the changes of ridership, in general, depend on the time distance, i.e., the time required for traveling between stops, and exhibit spatial scale invariance $[19,20]$.

Given such characteristics, we define the logarithmic change of ridership as

$\psi(\mathbf{r} ; t) \equiv \ln \left[\frac{s(\mathbf{r} ; t)}{s^{*}(\mathbf{r} ; t)}\right]$

where $s^{*}(\mathbf{r} ; t)$ and $s(\mathbf{r} ; t)$ are the expected ridership and the actual ridership, respectively, of the stop at location $\mathbf{r}$ on 
given day $t$. Expected ridership can be estimated in various ways: When probing fluctuations in 1-week data, one may take the time average of the data over 5 weekdays as an estimate of expected ridership. In case that sufficient data are available, one can take the average over the same days of the week to eliminate daily trends. If the data cover a year or longer, one can consider the ridership a year before or later.

To examine how the daily ridership evolved in time, we compute the logarithmic change in Eq. (1) for every stop in Seoul, from the ridership data of 2016. Taking the spatial average (i.e., average over all stops), we obtain the mean logarithmic change given by

$\mu_{t} \equiv\langle\psi(\mathbf{r} ; t)\rangle \equiv \frac{1}{N} \sum_{\mathbf{r}} \psi(\mathbf{r} ; t)$

and plot its time evolution in Fig. 2c, with error bars representing the first and the third quartiles. The time line of the behavior is disclosed well by the cross-correlation between the ridership change $\mu_{t}$ and the number $m_{1}(t)$ of new infections reported or the number $m_{2}(t)$ of Google searches. Specifically, it is measured using the Pearson correlation coefficient with time lag $\Delta t$ :

$\rho_{\mu, m_{k}}(\Delta t) \propto\left\langle\left(\mu_{t+\Delta t}-\bar{\mu}\right)\left(m_{k}(t)-\bar{m}_{k}\right)\right\rangle$,

where $\bar{\mu} \equiv\left\langle\mu_{t}\right\rangle$ and $\bar{m}_{k} \equiv\left\langle m_{k}(t)\right\rangle$ are the means of $\mu_{t}$ and $m_{k}(t)(k=1,2)$, respectively. Computing $\rho_{\mu, m_{k}}$ from Eq. (3), we find that the cross-correlation between the ridership and the number of case reports reaches its minimum at a time lag of zero while the minimum of the cross-correlation between the ridership and the search volume is located at a time lag of 4 days: $\rho_{\mu, m_{2}}(\Delta t) \rightarrow \min$ at $\Delta t=4$ days, which coincides with the downtrend lasting for 4 days. (Here the number of new infections and the search volume are given on a linear scale because both datasets include zeros.) This suggests that the behavior of the logarithmic change correctly describes the time line of the MERS outbreak. When only weekdays are considered, the mean logarithmic change began to decrease on 2 June, hit bottom on 11 June, and increased to zero eventually, meaning recovery to the level in the year 2016.

To quantify the duration of MERS effects on passenger behavior, we fit an exponential function

$\mu_{t}=-a e^{-t / \tau}$

to the data in the relaxation interval after the peak of anxiety. Considering also the possibility of power-law relaxation, we have attempted to fit an algebraic function and obtained a root-mean-square error of 0.098 , substantially larger than the value 0.058 for the exponential function. The relaxation, is thus, more consistent with an exponential one, albeit algebraic relaxation may not be decisively ruled out, characterized by the relaxation time $\tau=20.9 \pm 1.9$ days ( $\approx 3$ weeks $)$ and the amplitude $a=0.087 \pm 0.005$. Such an exponential decay may be interpreted as the effects of MERS decreasing at a rate proportional to its current amount.

To analyze the spatiotemporal relaxation of the ridership under the external perturbation caused by the MERS outbreak, we now focus on the period of 6 weeks between 8 June and 17 July. Figure 3 presents heatmaps, displaying the spatial distribution of logarithmic changes $\psi(\mathbf{r})$ given by Eq. (1) on 1 June and on 8 June. The color of each grid in the heatmap represents the mean logarithmic changes of bus stops within the grid. The size of each grid is $500 \mathrm{~m}$, which has been chosen in such a way that bus stops in each grid are within walking distance [44]. Note that in contrast with the heatmap 1 week earlier, the heatmap on 8 June is covered almost by blue grids. This manifests an overwhelming decrease in ridership over the entire region of Seoul.

Another feature is the spatial extent to which the logarithmic changes vary with each other. While changes of ridership at bus stops are coupled to each other, the bus routes and human mobility are finite. Accordingly, the logarithmic changes within some spatial extent should display the same tendency in variations. To visualize such spatial extent, we distinguish in Fig. 4 the grids with logarithmic changes below and above the mean value $\mu$ in the entire region of Seoul, which are colored cyan and magenta, respectively.
Fig. 3 Spatial distribution of the logarithmic changes in Seoul (a) on 1 June and (b) on 8 June. Each grid of size $500 \mathrm{~m}$ is colored according to the mean logarithmic changes of stops inside the grid

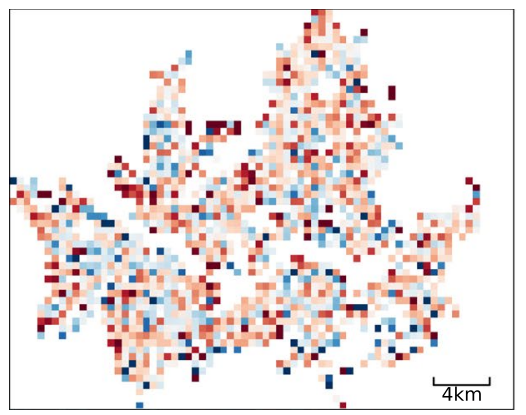

(a) 1 June

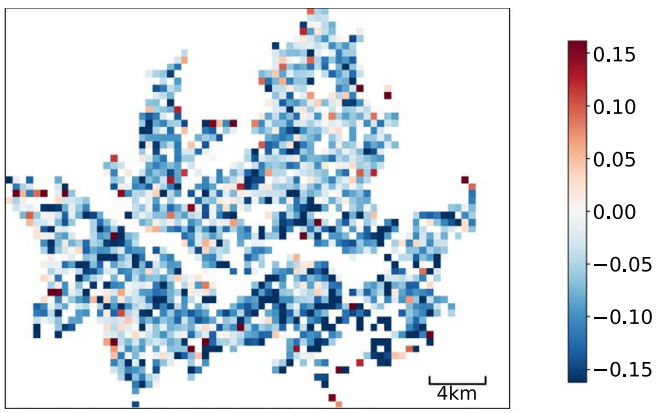

(b) 8 June 
Fig. 4 Spatial distribution of the logarithmic changes relative to the mean value (a) on 8 June and (b) on 15 June. The size of each grid is the same as that in Fig. 3. Grids with the logarithmic changes below and above the mean are colored cyan and magenta, respectively. The red solid circle represents SMC whereas black solid circles designate those hospitals included in the daily keyword list (a) before 8 June and (b) before 15 June

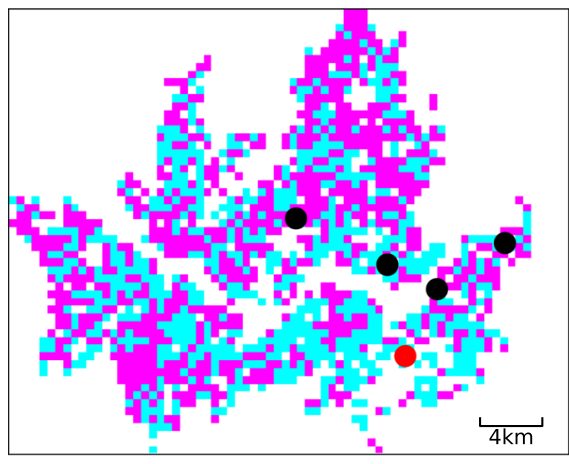

(a) 8 June

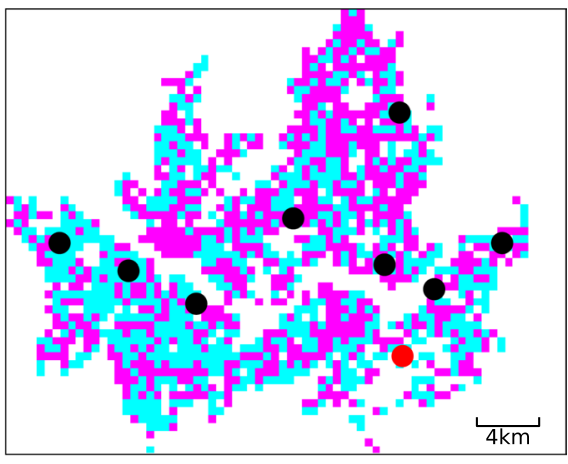

(b) 15 June
The large cluster of cyan grids, emerging with the MERS outbreak, manifests the enlargement of the spatial extent in which the logarithmic changes vary from each other. Such severe decreases were caused by the hospitals treating patients. The majority of infected patients were treated in a few hospitals, which attracted great attention. For instance, the large cyan cluster observed at the right bottom in Fig. 4a is attributed to Samsung Medical Center (SMC) (marked with a red solid circle), popularly known as the main source of infection. As additional hospitals were newly reported in the beginning of the recovery stage, the clusters kept modifying. The number of hospitals related to MERS reached more than 30 in Seoul. By analyzing the top 100 daily keywords in the 2 most popular search engines in Korea, Naver and Daum, we sorted and sequenced seven general hospitals. From 7 to 11 June, the hospitals were newly listed every day in the daily keywords. In Fig. 4a, b, these hospitals are designated by black solid circles if they were included in the daily keyword list before 8 June and before 15 June, respectively. Note that in contrast with (a), there are hospitals marked with circles on the left as well as at the right bottom of (b); this provides an explanation for the newly formed large cluster on the lower left.

\section{Formulation and data analysis}

In this section, we investigate the spatiotemporal changes of ridership in a framework based on linear response theory. Changes of ridership at different stops should be coupled with each other because passengers use at least two stops to ride and to alight. This gives rise to interesting collective behaviors in the spatial distribution of changes, which is manifested by the correlation function

$\Gamma\left(\mathbf{r}, \mathbf{r}^{\prime} ; t, t^{\prime}\right) \equiv \sigma_{t}^{-2}\left[\left\langle\psi(\mathbf{r} ; t) \psi\left(\mathbf{r}^{\prime} ; t^{\prime}\right)\right\rangle-\mu_{t} \mu_{t^{\prime}}\right]=\left\langle\tilde{\psi}(\mathbf{r} ; t) \tilde{\psi}\left(\mathbf{r}^{\prime} ; t^{\prime}\right)\right\rangle$ with the standard deviation $\sigma_{t} \equiv \sqrt{N^{-1} \sum_{\mathbf{r}}\left[\psi(\mathbf{r} ; t)-\mu_{t}\right]^{2}}$. Here, for convenience, we have defined the normalized change as

$\tilde{\psi}(\mathbf{r} ; t) \equiv \frac{\psi(\mathbf{r} ; t)-\mu_{t}}{\sigma_{t}}$,

which vanishes upon averaging: $\langle\tilde{\psi}(\mathbf{r} ; t)\rangle=0$.

We consider the system to have been essentially in equilibrium without any external perturbation, i.e., in the absence of the epidemic outbreak. In that case, there is no explicit time dependence; namely, the ridership does not vary appreciably day by day, which allows one to suppress the time variable $t$ in Eqs. (1), (2), (5), and (6). (See Appendix for the discussion on the case of a time-dependent perturbation.) By analogy with a physical system in statistical mechanics, we introduce formally a distribution function $P(\psi)$ and henceforth express $\langle\cdots\rangle$ as the (statistical) ensemble average with respect to $P(\psi)$. We further define the dimensionless "energy function" as $E(\psi) \equiv-\ln P(\psi)+C$, where $C$ is a constant ensuring the normalization of $P(\psi)$. This gives the distribution function in the canonical form $P(\psi)=Z^{-1} e^{-E(\psi)}$, where the "temperature" has been absorbed into the energy function. Obviously, the normalization constant $Z \equiv e^{-C}=\mathrm{T} r e^{-E(\psi)}$, where $\operatorname{Tr}$ stands for the summation over all configurations, i.e., integration with respect to all $\psi(\mathbf{r})$, corresponds to the partition function. Then, the outbreak of an epidemic is considered to have brought about an appreciable perturbation to the system, and we are interested in how the system responds to such an external perturbation.

Suppose that the perturbation $h(\mathbf{r})(>0)$ given at location $\mathbf{r}$ pulls the change $\psi(\mathbf{r})$ upward. This can conveniently be taken into account by introducing an additional term $-\sum_{\mathbf{r}} h(\mathbf{r}) \tilde{\psi}(\mathbf{r})$ to the energy function $E(\psi)$; such a linear term should be appropriate unless $h(\mathbf{r})$ or $\tilde{\psi}(\mathbf{r})$ is large. Then $\tilde{\psi}(\mathbf{r})$ acquires a non-vanishing average value in response to the perturbation $h(\mathbf{r})$ : 
$\langle\tilde{\psi}(\mathbf{r})\rangle=\sum_{\mathbf{r}^{\prime}} K\left(\mathbf{r}, \mathbf{r}^{\prime}\right) h\left(\mathbf{r}^{\prime}\right)$

with the linear response function

$K\left(\mathbf{r}, \mathbf{r}^{\prime}\right)=\left.\frac{\delta\langle\tilde{\psi}(\mathbf{r})\rangle}{\delta h\left(\mathbf{r}^{\prime}\right)}\right|_{h\left(\mathbf{r}^{\prime}\right)=0}=\left.\frac{\delta^{2} \ln Z}{\delta h\left(\mathbf{r}^{\prime}\right) \delta h(\mathbf{r})}\right|_{h\left(\mathbf{r}^{\prime}\right)=h(\mathbf{r})=0}$.

Showing that Eq. (8) leads to $K\left(\mathbf{r}, \mathbf{r}^{\prime}\right) \propto\left\langle\tilde{\psi}(\mathbf{r}) \tilde{\psi}\left(\mathbf{r}^{\prime}\right)\right\rangle_{0}$ is straightforward, where $\langle\cdots\rangle_{0}$ denotes the (equilibrium) average in the absence of the perturbation. Thus the linear response function is, up to a constant, equal to the correlation function:

$K\left(\mathbf{r}, \mathbf{r}^{\prime}\right) \propto \Gamma_{0}\left(\mathbf{r}, \mathbf{r}^{\prime}\right)$,

where the subscript 0 signifies the absence of a perturbation. Note that Eq. (9) is just a simple instance of the fluctuationdissipation theorem relating responses to the applied perturbation with equilibrium fluctuations in the absence of that perturbation [36]. In particular, it is rather general, involving neither the energy function $E(\psi)$ nor the perturbation $h(\mathbf{r}, t)$; this allows the response behavior to be probed without specifying the detailed nature of the system and the perturbation.

As described in Sect. 2, the Seoul bus transportation system is well developed and accommodates numerous stops populated densely and rather homogeneously over the whole area, which makes taking the continuum description with translational and orientational invariance reasonable. The translational invariance may not exist in some transportation systems $[45,46]$, but this is not the case for our dataset, where the passenger trips are diverse in character, spread out, and distributed rather well at all times of a day, thus not being restricted to regular commuting. Then, the summations in Eqs. (2) and (7) are understood as corresponding integrations whereas Eq. (9) reduces to $K(r) \propto \Gamma_{0}(r)$, with $r \equiv\left|\mathbf{r}-\mathbf{r}^{\prime}\right|$ being the distance.

In the previous section, the epidemic outbreak was shown to have induced a striking decrease in ridership, as manifested by the spatial distributions of the logarithmic changes. Particularly small values of the logarithmic change were observed to be localized in typical areas near the hospitals treating patients. Namely, the normalized logarithmic change dropped sharply near the hospital and rose to zero as the distance from the hospital was increased. Such features reflect the presence of a negative perturbation localized most conspicuously at SMC in Seoul; the effects of MERS were most severe and SMC became the hottest spot of infection.

To describe the stationary spatial behavior during the epidemic, we, thus, write the perturbation in the form

$h(\mathbf{r})=-h \delta\left(\mathbf{r}-\mathbf{r}_{c}\right)$,

where $h(>0)$ is the perturbation strength, $\delta(\cdots)$ is the Dirac delta function, and $\mathbf{r}_{c}$ is the location of SMC in Seoul. Plugging this into Eq. (7), we obtain $\langle\tilde{\psi}(\mathbf{r})\rangle=-h K\left(\mathbf{r}, \mathbf{r}_{c}\right) \propto-\Gamma_{0}\left(\mathbf{r}, \mathbf{r}_{c}\right)$,

where Eq. (9) has also been used. Namely, the response in the logarithmic change is described precisely by the equilibrium correlation function in the absence of the perturbation.

Because the unperturbed system is considered homogeneous, isotropy should exist around the location $\mathbf{r}_{c}$ of the perturbation. Accordingly, $\langle\tilde{\psi}(\mathbf{r})\rangle$ depends only on the distance from $\mathbf{r}_{c}$, so we may write $-\tilde{\psi}(r) \equiv\langle\tilde{\psi}(\mathbf{r})\rangle_{\left|\mathbf{r}-\mathbf{r}_{c}\right|=r}$, where $\langle\cdots\rangle_{\left|\mathbf{r}-\mathbf{r}_{c}\right|=r}$ denotes the average over all stops separated from $\mathbf{r}_{c}$ by distance $r$. Equation (11), thus, takes the simple form

$-\tilde{\psi}(r) \propto \Gamma_{0}(r)$,

where $r$ is the distance from SMC.

We first examine the correlation function between normalized logarithmic changes $\tilde{\psi}$, relating the ridership of different stops separated by distance $r$. To obtain the equilibrium correlation function, we consider a period before the MERS outbreak, specifically, weekdays from 10 May to 6 June (excluding one holiday). In contrast, weekdays from 6 to 20 June are selected as the rampant period, i.e., as the duration of the perturbation. For comparison, we also consider weekdays from 20 June to 4 July when the system apparently relaxed to equilibrium after the MERS outbreak. Computing the correlation function between each pair of stops and taking the average over the pairs separated by a given distance, we obtain the correlation functions for three periods and plot them in Fig. 5. In the figure, the data points are observed to display linear behavior on a log-log scale, indicating a power-law decay with distance $r$ :

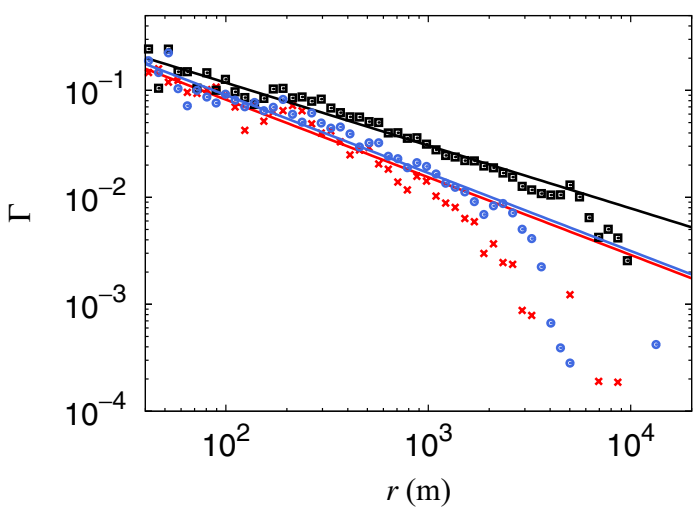

Fig. 5 Decay of the correlation function $\Gamma$ with distance $r$. Data points labeled by red crosses describe the correlation function before the outbreak of MERS (from 10 May to 6 June). Data points designated by black squares depict the function in the rampant period (from 6 to 20 June); those by blue circles represent the function after the outbreak (from 20 June to 4 July). The straight lines providing least-squares fits to the corresponding data and have slopes of 0.73 (red and blue) and 0.59 (black) 
$\Gamma(r) \sim r^{-\eta}$.

Attempts to use other functions such as an exponential one have yielded no better results, as judged via the root-meansquare errors. The least-squares fit to the data points for $r$ between $40 \mathrm{~m}$ and $4000 \mathrm{~m}$ allows us to estimate the exponent $\eta$. Specifically, the data for the period before the MERS outbreak give the equilibrium correlation function $\Gamma_{0}(r)$ decaying algebraically with exponent $\eta_{0}=0.73 \pm 0.03$. The data for the period from 20 June to 4 July also lead to essentially the same behavior, with exponent $\eta_{0}=0.73 \pm 0.05$, which indicates that the system, indeed, relaxed to stationarity. Of interest is the observation that the correlation function in the rampant period also displays an algebraic decay, but is clearly distinguished from the others in that the exponent is smaller: $\eta=0.59 \pm 0.04$. Note that a smaller $\eta$ means a slower decay, which is observed in case when the decrease in ridership is obvious.

We next probe how the logarithmic change varied with the distance from the hospital, which is expected to exhibit a substantial decrease in the immediate vicinity and to relax to the mean $\mu$ at large distance. We, thus, compute logarithmic changes for all stops in Seoul and take the average over all stops separated from $\mathbf{r}_{c}$ by distance $r$, which gives $\tilde{\psi}(r)$ as described above. Plotted in Fig. 6 are the data for $\tilde{\psi}(r)$ during 5 weekdays from 8 to 12 June, which belong to the duration of the perturbation, i.e., when MERS is rampant. Despite fluctuations of the data points, the overall behavior is mostly consistent with a power law behavior

$-\tilde{\psi} \sim r^{-\phi}$.

Again, fitting an exponential function results in a larger value of the root-mean-square error (i.e., 0.33 versus 0.18 ).

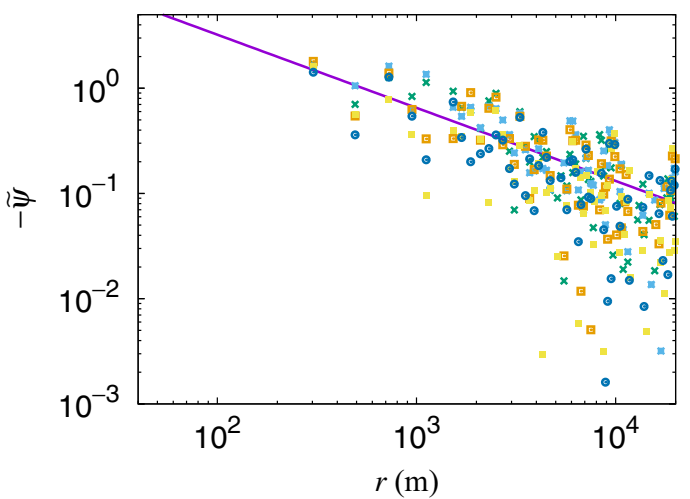

Fig. 6 Spatial relaxation of the normalized logarithmic change $\tilde{\psi}$ with the distance $r$ from Samsung Medical Center. Scatter plots of data points in the rampant period of 5 weekdays (8-12 June) are presented, together with the fitted line, on a logarithmic scale. The slope of the line is -0.69
Fitting the data points for $r$ below $4000 \mathrm{~m}$, we estimate the exponent $\phi=0.69 \pm 0.07$. Note that Eq. (12) requires $\phi$ to equal $\eta_{0}$ of the equilibrium correlation function $\Gamma_{0}(r)$. Indeed $\phi=0.69 \pm 0.07$ coincides with $\eta_{0}=0.73 \pm 0.03$ within the error bars, thus confirming the validity of the fluctuation-dissipation theorem.

\section{Conclusions}

Analyzing the Seoul bus ridership data during the MERS outbreak, we have studied the spatiotemporal behavior of a public transportation system under an external perturbation. The decrease in ridership caused by the MERS outbreak appeared 2 weeks after the official report of the first case. The relaxation of the decrease observed in all of Seoul has been found to be described by an exponential function with a characteristic time of 3 weeks, after which the correlation function also returned to the equilibrium one.

The spatial distribution of logarithmic changes manifests a severe decrease in areas close to the hospitals treating patients. We have arranged the hospitals in time order from the daily keyword data in popular search engines and identified that the spatiotemporal variations of the hospitals match with formations of severely decreased areas. Particularly, while the impact of MERS was most severe and SMC was the hottest spot of infection, the normalized logarithmic change dropped sharply near SMC, manifesting a localized perturbation at SMC, and rose to zero as the distance from the hospital was increased, being characterized by a power-law spatial relaxation. Such features have been probed through the use of the linear response theory. We consider the system to have been in equilibrium before the MERS outbreak and then to respond to the localized perturbation. According to the fluctuation-dissipation theorem, spatial relaxation should be described by the equilibrium correlation function. The equilibrium correlation function obtained from the data before the MERS outbreak has been observed to be characterized by a power law with an exponent coincident with that for spatial relaxation, thus indeed confirming the fluctuation-dissipation theorem.

Interestingly, the correlation function in the rampant period has also turned out to obey a power law, albeit the exponent is smaller. Here, the exponent of the power-law correlation function provides a measure of the degree of correlations. The smaller exponent during the rampant period reflects that the correlations decay more slowly compared with those in normal periods without MERS. This indicates that, during the rampant period, each bus stop or region of the city reacted acutely to the changes in other stops or regions even when they were far away. Namely, people became concerned about what happened in areas they did not normally care about. On the other hand, dynamic 
scaling is apparently lacking in these spatiotemporal relaxation behaviors, presumably due to the difference between the spatial and the temporal localization properties of the perturbation, although limitations in the temporal resolution of the data do not allow clear conclusions (see Appendix for further discussion).

In addition to the bus system, the subway system serves as another major transportation mode in Seoul, making consideration of the subway ridership desirable. Unlike bus stops, however, subway stations are sparse and unevenly distributed. In particular, subway routes often offer faster connections and long-distance shortcuts to the bus routes. To extend this research by considering the public transportation combined with bus and subway routes, we, thus, need to devise a distance measurement other than the Euclidean distance. Specifically, the time distance between two regions might be a good candidate on which the correlation depends, because the shortest path in the combined transportation system is determined by the travel time.

Recall that the ridership at each bus stop, adopted as the local order parameter, has revealed power-law correlations in the Seoul bus system. Such power-law correlations are regarded as the essential collective behavior of criticality, which is a characteristic of complexity without a typical length scale. One is not surprised to observe complexity here, as many studies have been devoted to the complexity in urban transportation systems [15, 19, 47-49]. In addition, other properties such as scale invariance of fluctuations as well as the relation between the network density exponent and the fractal dimension of the system were also reported [20]. Naturally, such features are the consequence of the couplings between bus stops or regions of the city. As is well known, the competition between order attained by couplings and disorder reflecting fluctuations or randomness is crucial for criticality. In the growth and fall of a city, these properties appear to be organized to generate the complex structure of the city [50]. These couplings are dictated by the travel behavior because passengers do not use bus or subway routes in a random way, but want to minimize the travel time between the origin and the destination. Understanding the couplings and fluctuations is essential for establishing theoretical models for transportation systems and cities, which is left for future study.

As for the outbreak of COVID-19, examining the affected ridership through the use of our model is certainly relevant. The Government of Korea first announced the seriousness of the pandemic on 14 February 2020. Subsequently, the number of passengers using the public transportation system in Seoul dropped by $38 \%$ compared to the previous year. As in the case of the MERS outbreak, the decrease was drastic, but the recovery was relatively gradual until the second stage of the pandemic. The drop at the peak of the second stage also turned out to be drastic, more than $30 \%$. Note that while COVID-19 is still ongoing, such ridership data are being actively accumulated. Moreover, unlike the case of MERS, the outbreak of COVID-19 has occurred at diverse places in addition to hospitals. In other words, it is not localized but global, spreading not only in Seoul but throughout Korea and the world. Thus, examining the responses to such delocalized perturbations in analyzing the public transportation data when COVID-19 is brought under control and stabilized would be of interest. We expect that the present study of the MERS outbreak should provide a useful guide to such a study of the pandemic.

These results should not only deepen our fundamental understanding of human dynamics but also improve emergency responses. While aid organizations increasingly use distributed, real-time communication tools in the 21st century, disaster research often continues to rely on low-throughput, post-event data, such as questionnaires, eyewitness reports, and communication records between responders and relief organizations. The emergency case explored here indicates that collective changes in human activity patterns can be captured objectively and quantitatively. In addition, this study improves the understanding of the urban transportation system, which is essential for probing principles of social systems because a majority of people reside in urban areas in this modern age and most urban flows are realized through mass transportation system such as subways or bus networks.

Acknowledgements One of us (M.Y.C.) acknowledges the support from the National Research Foundation through the Basic Science Research Program (Grant No. 2019R1F1A1046285).

\section{Appendix}

To probe the relaxation behavior after the epidemic is over, we further consider a time-dependent perturbation $h(\mathbf{r}, t)$, for which the standard linear response theory leads Eq. (7) to assume the general form $[34,35,37]$

$\langle\tilde{\psi}(\mathbf{r} ; t)\rangle=\sum_{\mathbf{r}^{\prime}} \int_{-\infty}^{t} d t^{\prime} K\left(\mathbf{r}, \mathbf{r}^{\prime} ; t, t^{\prime}\right) h\left(\mathbf{r}^{\prime}, t^{\prime}\right)$,

with the linear response function

$$
\begin{aligned}
& K\left(\mathbf{r}, \mathbf{r}^{\prime} ; t, t^{\prime}\right)=\left.\frac{\delta\langle\tilde{\psi}(\mathbf{r} ; t)\rangle}{\delta h\left(\mathbf{r}^{\prime}, t^{\prime}\right)}\right|_{h\left(\mathbf{r}^{\prime}, t^{\prime}\right)=0} \\
& \quad \propto\left\langle\tilde{\psi}(\mathbf{r} ; t) \tilde{\psi}\left(\mathbf{r}^{\prime} ; t^{\prime}\right)\right\rangle_{0} \propto \Gamma_{0}\left(\mathbf{r}, \mathbf{r}^{\prime} ; t, t^{\prime}\right) .
\end{aligned}
$$

Specifically, in case that the perturbation $h\left(\mathbf{r}^{\prime}\right)$ is turned off at time $t^{\prime}=0$, Eq. (15) for $t>0$ reduces to

$\langle\tilde{\psi}(\mathbf{r} ; t)\rangle=\sum_{\mathbf{r}^{\prime}} R\left(\mathbf{r}, \mathbf{r}^{\prime} ; t\right) h\left(\mathbf{r}^{\prime}\right)$,

where $R\left(\mathbf{r}, \mathbf{r}^{\prime} ; t\right)$ is the relaxation function given by $[34,37]$ 
$R\left(\mathbf{r}, \mathbf{r}^{\prime} ; t\right)=\int_{t}^{\infty} d \tau K\left(\mathbf{r}, \mathbf{r}^{\prime} ; \tau, 0\right) \propto \Gamma_{0}\left(\mathbf{r}, \mathbf{r}^{\prime} ; t, 0\right)$.

Now, we consider the temporal relaxation after the peak of anxiety, i.e., the response to turning off the perturbation. For instance, we may take the time-dependent perturbation in the simple form $h(\mathbf{r}, t)=-h \delta\left(\mathbf{r}-\mathbf{r}_{c}\right) \theta(-t)$, where $\theta(t)$ is the Heaviside step function and $\mathbf{r}_{c}$ the location of SMC. Then reducing Eq. (17) to the form

$\langle\tilde{\psi}(\mathbf{r} ; t)\rangle=-h R\left(\mathbf{r}, \mathbf{r}_{c} ; t\right) \propto-\Gamma_{0}\left(\mathbf{r}, \mathbf{r}_{c} ; t, 0\right)$,

which indicates that the relaxation is directly proportional to the retarded correlation function, is straightforward. However, obviously, only one MERS outbreak occurred in Seoul. Namely, there is only one data set or one member of the ensemble, disallowing a statistical (ensemble) average. Without the ensemble averages taken, Eq. (19), upon averaging over all stops $\mathbf{r}$, reduces to a self-evident relation, which makes it irrelevant to check whether the temporal relaxation and the retarded correlation exhibit the same behaviors as implied by Eq. (19).

\section{References}

1. J.-P. Rodrigue, C. Comtois, and B. Slack, The Geography of Transport Systems ( Routledge, New York, 2016)

2. A. Mangili, M.A. Gendreau, Lancet 365, 989 (2005)

3. M. Brahmbhatt, A. Dutta, On SARS Type Economic Effects during Infectious Disease Outbreaks ( The World Bank (Washington D.C., 2008)

4. E.P. Fenichel, N.V. Kuminoff, G. Chowell, PLoS ONE 8, e58249 (2013)

5. L.M. Marafa, F. Tung, World Leis. J. 46, 38 (2004)

6. C.M. Peak et al., Int. J. Epidemiol. 47, 1562 (2018)

7. G. Nian, B. Peng, D.J. Sun, W. Ma, B. Peng, T. Huang, Sustainability 12(2020)

8. S. Eubank, H. Guclu, V.A. Kumar, M.V. Marathe, A. Srinivasan, Z. Toroczkai, N. Wang, Nature 429, 180 (2004)

9. S. Funk, E. Gilad, C. Watkins, V.A. Jansen, Proc. Natl. Acad. Sci. U.S.A. 106, 6872 (2009)

10. S. Funk, M. Salathé, V.A. Jansen, J. Roy. Soc. Interface 7, 1247 (2010)

11. S. Meloni, N. Perra, A. Arenas, S. Gómez, Y. Moreno, A. Vespignani, Sci. Rep. 1, 62 (2011)

12. L. Hufnagel, D. Brockmann, T. Geisel, Proc. Natl. Acad. Sci. U.S.A. 101, $15124(2004)$

13. V. Colizza, A. Barrat, M. Barthélemy, A. Vespignani, Proc. Natl. Acad. Sci. U.S.A. 103, 2015 (2006)

14. D. Brockmann, L. Hufnagel, T. Geisel, Nature 439, 462 (2006)

15. K. Lee, W.-S. Jung, J.S. Park, M.Y. Choi, Physica A 387, 6231 (2008)

16. M.C. Gonzalez, C.A. Hidalgo, A.-L. Barabasi, Nature 453, 779 (2008)

17. K. Lee, J.S. Park, H. Choi, W.-S. Jung, M.Y. Choi, J. Korean Phys. Soc. 57, 823 (2010)

18. C. Song, Z. Qu, N. Blumm, A.-L. Barabási, Science 327, 1018 (2010)
19. S. Goh, K. Lee, J.S. Park, M.Y. Choi, Phys. Rev. E 86, 026102 (2012)

20. S. Goh, K. Lee, M.Y. Choi, J.-Y. Fortin, PLoS ONE 9, e89980 (2014)

21. J.-H. Lee, J.W. Kim, K. Lee, M.Y. Choi, EPL 132, 48001 (2020)

22. J.C. Bohorquez, S. Gourley, A.R. Dixon, M. Spagat, N.F. Johnson, Nature 462, 911 (2009)

23. D. Balcan, V. Colizza, B. Gonçalves, H. Hu, J.J. Ramasco, A. Vespignani, Proc. Natl. Acad. Sci. U.S.A. 106, 21484 (2009)

24. J.P. Bagrow, D. Wang, A.-L. Barabasi, PLoS ONE 6, e17680 (2011)

25. L. Gao, C. Song, Z. Gao, A.-L. Barabási, J.P. Bagrow, D. Wang, Sci. Rep. 4, 3997 (2014)

26. W.-J. Kim, J. Korean Soc. Info. Manag 32, 273 (2015)

27. I.-H. Kim, C.-C. Feng, Y.-C. Wang, B.H. Spitzberg, M.-H. Tsou, Prof. Geogr. 69, 629 (2017)

28. P. Durai, M. Batool, M. Shah, S. Choi, Exp. Mol. Med. 47, e181 (2015)

29. MERS Effect on the Tourism Industry, http://www.kiet.re.kr/kiet_ web/?sub_num $=9 \&$ state $=$ view $\& i d x=51116$, Accessed 28 July 2021

30. H. Jung, M. Park, K. Hong, E. Hyun, Sustainability 8, 454 (2016)

31. H. Sung, M. Kwak, J. Korea Plan. Assoc. 51, 165 (2016)

32. D. Hanna, Y. Huang, Asian Econ. Pap. 3, 102 (2004)

33. H. Sung, J. Korea Plan. Assoc. 51, 163 (2016)

34. S. W. Lovesey, Condensed Matter Physics: Dynamic Correlations ( Benjamin/Cummings, Menlo Park, 1986)

35. R. Zwanzig, Nonequilibrium Statistical Mechanics (Oxford University Press, Oxford, 2001)

36. U. Marconi, A. Puglisi, L. Rondoni, A. Vulpiani, Phys. Rep. 461, $111(2008)$

37. N. Pottier, Nonequilibrium Statistical Physics: Linear Irreversible Processes (Oxford University Press, Oxford, 2009)

38. Modal Share, http://news.seoul.go.kr/traffic/archives/289, Acces sed 28 July 2021

39. Ridership Database, http://data.seoul.go.kr/dataList/OA-12913/S/ 1/datasetView.do, Accessed 28 July 2021

40. R. de Regt, C. von Ferber, Y. Holovatch, M. Lebovka, TRANSPORTMETRICA A 15, 722 (2019)

41. Daily number of new MERS infections (the Ministry of Health and Welfare repository), http://www.mohw.go.kr/react/al/sal03 011s.jsp?PAR_MENU_ID $=0403 \&$ BOARD_ID $=140 \&$ SEARC HKEY $=$ TITLE \&SEARCHVALUE $=\% \mathrm{~EB} \%$ A9\% $94 \% \mathrm{~EB} \% \mathrm{~A} 5 \%$ B4\%EC\%8A\%A4\&page=1, Accessed 28 July 2021

42. Google search volume (Google Trends) with the search term "MERS" (in Korean), https://trends.google.co.kr/trends/explore? date $=2015-05-09 \% 202015-09-12 \&$ geo $=$ KR\&q $=\%$ EB $\%$ A9\% $94 \%$ EB\%A5\%B4\%EC\%8A\%A4, Accessed 28 July 2021

43. K. Lee, S. Goh, J.S. Park, W.-S. Jung, M.Y. Choi, J. Phys. A 44, 115007 (2011)

44. M.H. Lee, J.H. Lee, H.S. Won, E.Y. Shin, Seoul Stud 20, 79 (2019)

45. M. Kurant, P. Thiran, Phys. Rev. E 74, 036114 (2006)

46. M.M. Izawa, F.A. Oliveira, D.O. Cajueiro, B.A. Mello, Phys. Rev. E 96, 012309 (2017)

47. M. Batty, Y. Xie, Discrete Dyn. Nat. Soc. 3, 109 (1999)

48. Y. Chen, Y. Zhou, Chaos Soliton Fract. 35, 85 (2008)

49. G. Petri, P. Expert, H.J. Jensen, J.W. Polak, Sci. Rep. 3, 1798 (2013)

50. S. Goh, M.Y. Choi, K. Lee, K.-M. Kim, Phys. Rev. E 93, 052309 (2016)

Publisher's Note Springer Nature remains neutral with regard to jurisdictional claims in published maps and institutional affiliations. 